\title{
Treatment of Refractory Status Epilepticus: Better Evidence is Needed
}

Keywords: Evidence, isoflurane, refractory status epileptics

doi:10.1017/cjn.2015.15

Can J Neurol Sci. 2015; 42: 72-73

Status epilepticus (SE) signifies the brain's failure to terminate seizures. This relates to excitatory mechanisms overwhelming inhibitory systems. ${ }^{1}$ Untreated, generalized convulsive SE leads to neuronal damage or death, the latter related to systemic complications, with neuronal damage related primarily to excitotoxicity. $^{2}$ Nonconvulsive seizures, except for absence status, can also cause neuronal death, especially in the hippocampus. ${ }^{3-5}$ The time for convulsive seizures to begin causing neuronal death is about 30 minutes, while with nonconvulsive seizures the time is longer, probably an hour or more. ${ }^{6}$ The longer the seizure persists the harder it is to stop as there is a progression of the excitation and further diminution of inhibition.

Thus, the brain needs help in arresting SE. The literature provides only limited evidence-based assistance in this regard. Intravenous lorazepam and intramuscular midazolam have been shown to be effective in the initial treatment of early status epilepticus in randomized controlled trials ${ }^{7-9}$ The evidence for the effectiveness of other agents is not of good quality. However, intravenous valproate and phenytoin are effective in stabilizing patients with SE. ${ }^{10,11}$ Published guidelines, based on expert opinion, recommend that an anesthetic agent be used if seizures continue. ${ }^{12,13}$

Seizures that require more than two first line drugs over the first hour are referred to as refractory status epilepticus (RSE) and those that persist for 24 hours or more despite treatment with an anesthetic agent are termed super-refractory status epilepticus (SRSE). ${ }^{14}$

As Zeiler and colleagues have pointed out in their comprehensive review of the role of inhalational agents (IAs) for RSE (really SRSE) in this issue, the quality of evidence for the effectiveness of inhalational agents is really quite poor (level D). ${ }^{15}$ However, since RSE and SRSE are unlikely to stop by themselves, unless the brain is largely destroyed, any association of the agent and seizure cessation should be taken seriously as support for effectiveness. IAs, notably isoflurane, can rapidly induce a burst-suppression and can temporarily stop seizure discharges. A problem that we and others have encountered is that seizures often recur when the anesthetic agent is stopped. This is likely "the nature of the beast" as reflected in Shorvon's classification of $\mathrm{SRSE}^{14}$ and the observation that longer duration seizures are more difficult to stop.

While there is some evidence for SRSE responding to IAs, mortality and morbidity, especially for neurological impairment, are still high. ${ }^{15}$ Also, there is a very high rate of treatment-related complications, including hypotension, paralytic ileus, superinfections, atelectasis and deep venous thrombosis. ${ }^{16}$ It has been difficult to tease out the relative contributions of etiology (e.g., encephalitis) and the seizures in cases of SE, RSE or SRSE that lead to the frequently encountered poor outcomes. ${ }^{17}$ Another factor that has been insufficiently considered for IAs is their potential neurotoxicity. Experimental models have revealed that isoflurane has the potential to be both neuroprotective and neurotoxic. ${ }^{18}$ Longer duration of treatment and higher concentrations of halothane exposure predisposes to apoptosis, while lower concentrations appear to be neuroprotective. ${ }^{18}$ The former conditions may apply in the application of isoflurane in the treatment of SRSE. Neurotoxicity with isoflurane has been suggested with the observation of reversible magnetic resonance imaging changes in the thalami, medulla, cerebellum and hypothalamus that appeared during treatment with isoflurane. ${ }^{19}$ We have also observed signal change in the hippocampus occurring more often in patients with SRSE treated with isoflurane, ${ }^{20}$ but we are in the process of validating these results. Other than these observations, the evidence for neurotoxicity in humans is meager. There are additional concerns for very young children. ${ }^{18}$

In summary, there is only Class D evidence for a role for inhalational agents, especially isoflurane and desflurane in the management of RSE and SRSE. The agents can stop electrographic seizures at least during administration, but seizures often recur when IA is withdrawn in SRSE cases. Concerns remain regarding complications of treatment with isoflurane at high concentrations for prolonged treatment times. Further research is clearly needed. It seems axiomatic that outcomes are best if seizures can be stopped early and completely in their course without compromising or causing harm to the patient. Such an ideal has yet to be accomplished.

\section{Disclosures}

The authors have nothing to declare.

G. Bryan Young, Kristin M. Ikeda
Department of Clinical Neurological Sciences, Western
University, London, Ontario, Canada
bryan.young@Ihsc.on.ca

\section{REFERENCES}

1. Hunter G, Young GB. Status epilepticus: a review with emphasis on refractory cases. Can J Neurol Sci. 2012;39:157-69.

2. Meldrum B. Excitotoxicity and epileptic brain damage. Epilepsy Res. 1991;10:55-6.

3. Ferguson M, Bianchi MT, Sutter R, et al. Calculating the Risk Benefit Equation for Aggressive Treatment of Non-convulsive Status Epilepticus. Neurocrit Care. 2013;18:216-27.

4. Young GB, Jordan KG. Do nonconvulsive seizures damage the brain?-Yes. Arch Neurol. 1998;55:117-9.

5. Vespa PM, McArthur DL, Xu Y, et al. Nonconvulsive seizures after traumatic brain injury are associated with hippocampal atrophy. Neurology. 2010;75:792-8. 
6. Lothman EW. The biochemical basis and pathophysiology of status epilepticus. Neurology. 1990;40(Suppl 2):13-23.

7. Treiman DM, Meyers PD, Walton NY, et al. A comparison of four treatments for generalized convulsive status epilepticus. N Eng J Med. 1998;339:792-8.

8. Alldredge BK, Gelb AM, Isaacs SM, et al. A comparison of lorazepam, diazepam, and placebo for the treatment of out-of hospital status epilepticus. N Engl J Med. 2001;345:631-7.

9. Silbergleit R, Durkalski V, Lowenstein D, et al. Intramuscular versus intravenous therapy for prehospital status epileptus. N Eng J Med. 2012;366:591-600.

10. Misra UK, Kalita J, Patel R. Sodium valproate vs. phenytoin in status epilepticus: a pilot study. Neurology. 2006;67:340-2.

11. Walker MC, Smith SJ, Shorvon SD. The intensive care treatment of convulsive status epilepticus in the UK: results of a national survey and recommendations. Anaesthesia. 1995;50:130-5.

12. Minicucci F, Muscas G, Perucca E, et al. Treatment of status epilepticus in adults: guidelines of the Italian League against Epilepsy. Epilepsia. 2006;47(Suppl 5):9-15.

13. Meierkord H, Boon P, Engelsen B, et al. EFNS guideline on the management of status epilepticus in adults. Eur J Neurol. 2010;17:348-55.
14. Shorvon S, Ferlisi M. The treatment of super-refractory status epilepticus: a critical review of available therapies and a clinical treatment protocol. Brain. 2011;134:2801-11.

15. Zeiler FA, Zeiler J, Teitelbaum J, et al. Modern inhalational anesthetics for refractory status epilepticus. Can J Neurol Sci. 2015; in this issue. doi:10.1017/cjn.2014.121.

16. Mirsattari S, Sharpe MD, Young GB. Treatment of refractory status epilepticus with inhalational anesthetic agents isofulrane and desflurane. Arch Neurol. 2004;61:1254-9.

17. Neligan A, Shorvon SD. Prognostic factors, morbidity and mortality in tonic-clonic status epilepticus: a review. Epilepsy Res. 2011;93:1-10.

18. Zuo Z. Are volatile anesthetics neuroprotective or neurotoxic. Med Gas Res. 2012;2:10.

19. Fugate JE, Burns JD, Wijdicks EFM, Warner DO, Jankowski CJ, Rabinstein A. Prolonged high-dose isoflurane for refractory status epilepticus: is it safe? Anesth Analg. 2010;111:1520-4.

20. Ikeda KM, Connors R, Claassen J, Young GB. MRI changes in refractory status epilepticus: Is isoflurane neurotoxic? Can $\mathrm{J}$ Neurol Sci. 2013;40(Suppl 1):S20. 